\title{
DISCONTINUOUS BOUNDARY CONDITIONS AND THE DIRICHLET PROBLEM*
}

BY

\author{
NORBERT WIENER
}

It has been suggested that in the Dirichlet problem there is something essentially antagonistic between the utmost degree of generality attainable as regards the geometrical character of the boundary and the utmost of generality attainable as regards the boundary values assumed over this boundary. The purpose of this paper is to show that the solution of the Dirichlet problem merely for continuous boundary conditions at once entrains the unique determination of a harmonic function correlated with discontinuous boundary conditions of a very general sort. The logical tool employed to this end is the Daniell integral.t In the stress laid on generalized types of integration this paper is closely akin to one of G. C. Evans, $\ddagger$ but it appears to the author that though the theory of Evans gives more detailed information concerning the character of the solutions of the Dirichlet problem in the neighborhood of the boundary, it is less direct than the present theory, and less extensible to regions of infinite connectivity or higher dimensionality.

\section{The POTential at a POINT as a linear functional OF THE BOUNDARY CONDITIONS}

Let $R$ be any open set of points in $n$-space connected in the sense that any two of its points form extremities of a polygonal line lying entirely within it, and not extending to infinity. Let the Dirichlet problem be solvable over $R$ for any continuous boundary cenditions on $C$. That is, if $U(P)$ is defined for everv point $P$ on $C$, and if

$$
\lim _{\overline{P Q} \rightarrow 0} U(P)=U(Q)
$$

\footnotetext{
* Presented to the Society, April 28, 1923.

$\dagger$ A general form of integral, Aunals of Mathemat1cs, ser. 2, vol. 19 (1918), p. 279.

$\mp$ Problems of potential theory, Proceedings of the National Academy of Sciences, vol. 7 (1921), pp. 89-98.
} 
for every $Q$ on $C$, then there is a function $u(P)$, defined and continuous over $R+C$, harmonic over $R$, and reducing to $U$ on $C$. With the aid of a form of generalized integral due to Daniell, we shall associate with any discontinuous function $V(P)$ of a very wide class of functions defined over $C$ a unique function $v(P)$ defined and harmonic over $R$, which we might naturally call the solution of the boundary ralue problem corresponding to $V(P)$.

Let $Q$ be a given point in the interior of $R$. Then $u(Q)$ may be regarded as a functional of $U(P)$. To symbolize this point of view, let us write

$$
I_{Q}(U)=u(Q)
$$

Since the functions harmonic over $R$ form a linear set. we have

$$
I_{Q}(C U)=C I_{Q}(U),
$$

and

$$
I_{Q}\left(U_{1}+U_{\mathbf{2}}\right)=I_{Q}\left(U_{1}\right)+I_{Q}\left(U_{\mathbf{2}}\right) .
$$

Moreover, because of the fact that no function harmonic over $R$ has extrema over $R$,

$$
I_{Q}(U) \geq 0 \text { if } U \geqq 0 .
$$

Another property of $I_{Q}$ is that

(L) If $U_{1}, U_{2}, \ldots U_{n}, \ldots$ is a sequence of continuous functions defined over $C$. and

$$
U_{1} \geqq U_{9} \geqq \cdots \geqq 0=\lim U_{n}
$$

for every point on $C$. then

$$
\lim I_{Q}\left(U_{n}\right)=0 .
$$

To prove this, it is sufficient to show that

$$
\lim \max U_{n}=0,
$$

since

$$
I_{Q}\left(U_{n}\right) \leqq \max U_{n} .
$$


If (1) were false, it would be possible to find a positive number $\varepsilon$ and a sequence $\left\{P_{n}\right\}$ of points on $C$ such that for every $n$

$$
U_{n}\left(P_{n}\right)>\varepsilon
$$

This sequence will have at least one limit element $N$, which will belong to $C$, as $C$ is a closed, bounded set. By hypothesis

$$
U_{1}(N) \geqq U_{2}(N) \geqq \cdots \geqq 0=\lim U_{n}(N)
$$

Hence there is a value of $n$, say $\nu$, such that

$$
U_{v}(N)<\varepsilon / 2 \text {. }
$$

Let $\left\{N_{n}\right\}$ be a sequence selected from the $P_{n}$ 's and approaching $N$ as a limit. It follows from the hypothesis of $(L)$ that for every $N_{n b}$ from some stage $n=\mu$ on

$$
I_{r}\left(N_{n}\right)>\varepsilon .
$$

Hence, since $U_{v}$, is continuous,

$$
U_{,}(N) \geqq \varepsilon .
$$

Since formulas (2) and (3) contradict one another, (1) and hence $(L)$ is proved The continuous functions defined over $C^{\gamma}$ form a linear set: that is, the sum of any two or a constant multiple of any one is also continuous. The absolute value of a continuous function is continuous. A continuous function is bounded over $C$. These properties of continuous functions are those attributed to a class $T_{0}$ by Daniell in the paper to which we have already referred. Moreover $\left(C^{r}\right),(A),(P)$, and $(L)$ show that the operator $I_{Q}$ fulfils the conditions which he has laid down for an operator $I$ on $T_{0}$. We are hence in a position to employ those definitions and theorems by which he enlarges the scope of the operator 1 .

\section{II. 'The DANiell EXTENSiON of $I_{Q}$}

In accordance with a definition of Daniell, a function $U$ is of the class $T_{1}$ if $U_{1} \leqq U_{2} \leqq \cdots$ is a non-decreasing sequence of functions from $T_{0}$ and $U=\lim U_{n}$. Under these circumstances, by a theorem of Daniell,

$$
I_{Q}\left(U_{1}\right)<I_{Q}\left(U_{2}\right) \leqslant \cdots,
$$


and either $\lim I_{Q}\left(U_{n}\right)$ exists or else $I_{Q}\left(U_{n}\right)$ becomes infinite. We shall write

$$
u(Q)=I_{Q}(U)=\lim I_{Q}\left(U_{n}\right)=\lim u_{n}(Q) .
$$

In general, we shall preserve the usage here indicated for the correlation of corresponding small and capital letters, applying the latter to functions on $C$, the former to the functions thereby determined as generalized integrals on $R$.

Now $u_{n}(Q)$ is harmonic over $R$. Hence the sequence $\left\{u_{n}\right\}$ is a monotone sequence of harmonic functions. It follows from a theorem of Harnack* that if $u(Q)$ is finite for any $Q$ in $R$, it is finite and harmonic for every $Q$ in $R$, while $u_{n}(Q)$ converges uniformly to $u(Q)$ over any closed region $S$ interior to $R$.

In accordance with Daniell's definition, if $V$ is any function defined on $C$, we shall write $I_{Q}(V)$ for the lower bound of $I_{Q}(U)$ for all the functions $U$ of class $T_{1}$ such that $U \geqq V$. Similarly, we make the definition

$$
I_{Q}(\nabla)=-\dot{I}_{Q}(-V) .
$$

When we have $I_{Q}(V)=\dot{I}_{Q}(V)$, we say that $V$ is summable, and with Daniell write what is in our notation

$$
v(Q)=I_{Q}(V)=I_{Q}(V)=\dot{I}_{Q}(V) .
$$

When $V$ is summable, it is clearly possible to find, whatever positive number $\varepsilon$ may be, a pair of functions $U_{1}$ and $U_{2}$, such that $U_{1}$ and $-U_{2}$ belong to $T_{1}$, while

$$
U_{1} \geqq V \geqq U_{\mathbf{2}},
$$

and

$$
I_{Q}\left(U_{1}\right)+I_{Q}\left(-U_{2}\right)=u_{1}(Q)-u_{2}(Q)<\varepsilon .
$$

The function $u_{1}(P)-u_{2}(P)$ is clearly harmonic. We now proceed as in the proof of Harnack's theorem. ${ }^{\dagger}$ Let us describe about $Q$ any circle lying entirely within $R$. Let $u_{1}-u_{z}$ assume the values $W$ on the periphery of this circle, the radius of which we take to be $a$. Then over the interior of the circle

* Osgood, Lehrbuch der Funktionentheorie, Leipzig, 1907, p.615. This theorem is there proved for the two-dimensional case, but the proof may be extended at once to $n$ dimensions. The restriction of the theorem to "Bereiche $S$ " is not essential, as it applies at once to all connected open regions.

† The proof is here given in the form appropriate to a space of two dimensions, but mutatis mutandis is valid in $n$ dimensions. For the sake of explicitness the language of two dimensions is used in several subsequent passages. 


$$
u_{1}-u_{2}=\frac{1}{2 \pi} \int_{0}^{2 \pi} W \frac{a^{2}-r^{2}}{a^{2}-2 a r \cos (\theta-\psi)+r^{2}} d \psi
$$

Since

$$
0 \leqq \frac{a^{2}-r^{2}}{a^{2}-2 a r \cos (\theta-\psi)+r^{2}} \leqq \frac{a+r}{a-r} \quad(r<a)
$$

we have

$$
\begin{aligned}
u_{1}-u_{2} & \leqq \frac{1}{2 \pi} \int_{0}^{2 \pi} W \frac{a+r}{a-r} d \psi \\
& =\left\{u_{1}(Q)-u_{2}(Q)\right\} \frac{a+r}{a-r} \\
& <\varepsilon \frac{a+r}{a-r} .
\end{aligned}
$$

It follows that within any circle about $Q$ or raaus less than $a$, by making $\varepsilon$ approach 0 , we shall make $u_{1}-u_{2}$ approach 0 uniformly. Since $u_{1}(P) \geqq \dot{I}_{P}(V)$ and $\iota_{2}(P) \leqq I_{P}(V)$, it follows that within this circle $v(P)$ exists, and is approached uniformly by $u_{1}(P)$ and $u_{2}(P)$ as $\varepsilon$ approaches 0 . Hence within this circle $v$ is harmonic. By the formation of chains of circles such as those used in the theory of analytic continuation, it may be shown that if $V$ is summable with regard to one point of $R$, it is summable with regard to all points of $R$, and that the function $v(P)$ thus defined is harmonic everywhere in $R$.

Daniell shows that the set of all summable functions is closed with regard to the operations of addition, multiplication by a constant, and taking the absolute value. He also shows that the extended operation $I_{Q}$ satisfies $(C),(A)$, and $(P)$. Condition $(L)$ is replaced by the important theorem which reads in our terminology as follows: if $\left\{U_{n}\right\}$ is a sequence of summable boundary functions such that $\lim U_{n}=U$, and if there is a summable function $V$ such that over all $C$ we have $\left|U_{n}\right| \leq \Sigma$ for all $n$, then $U$ is summable, and $u(P)$ $=\lim u_{n}(P)$. As one consequence of these theorems, functions belonging to $T_{0}$ and $T_{1}$ are summable, and all the definitions given of $I_{Q}(U)$ agree when more than one of them is applicable.

III. THE BEHAVIOR OF $I_{Q}(U)$ IN THE NEIGHBORHOOD OF THE BOUNDARY

If $Q$ is a point of $C$, and $K$ is a circle with $Q$ as center, then any function $U(P)$ which is defined and summable on $C$, bounded, and 0 over that part of $C$ 
within $K$, determines a harmonic function $u(P)$ which approaches 0 as $P$ approaches $Q$. To prove this, let us take $a$ as the radius of $K$, and let $K^{\prime}$ be a concentric circle with radius $a / 2$. Let the upper bound of $|U|$ be $M$. Then we shall define the function $V(P)$ as $M$ outside of $K, 0$ within $K^{\prime}$, and $M((2 r / a)-1)$ at those points in the annulus between $K$ and $K^{\prime}$ at a distance $r$ from $Q$. Clearly $V$ is continuous. The boundary values $V$ on $C$ determine a function $v(P)$ continuous on $R+C$, harmonic on $R$, and reducing to $V$ on $C$. By definition

$$
|U(P)| \leqq \nabla(P)
$$

over $C$. Hence by a theorem of Daniell

$$
|u(P)| \leqq I_{P}(|U|) \leqq v(P)
$$

This holds over all $k$. Since

$$
\lim _{P \rightarrow Q} v(P)=0=V(Q)
$$

we have

$$
\lim _{P \rightarrow Q} u(P)=0=U(Q)
$$

This result is capable of immediate generalization. In the first place, by the addition of a constant to $U$, we get the result that if $W(P)$ is a bounded function summable on $C$ and constant over $K$,

$$
\lim _{P \rightarrow \ell} w(P)=W(Q)
$$

Next let $W_{1}$ be any bounded summable function defined over $C$, and let $A$ and $B$ be respectively the upper and lower bounds of the oscillation of $W_{1}$ in the neighborhood of the point $Q$ of $C$. Then given any positive quantity $\varepsilon$, it is possible to describe a circle $K$ about $Q$ as center within which $A+\varepsilon>W_{1}(P)$ $>B-\varepsilon$. It then follows from the extension of $(P)$ to all summable functions and from (6) that if the functions formed from $W_{1}$ by substituting $A+\varepsilon$ or $B-\varepsilon$ within $K$ are summable, the oscillation of $v_{1}(P)$ in the neighborhood of $Q$ is not greater than from $A+\varepsilon$ to $B-\varepsilon$, and hence, since $\varepsilon$ is arbitrary, is not greater than from $A$ to $B$. If in particriar $W_{1}$ is continuous at $Q$, then the function which is $W_{1}$ on $C$ and $w_{1}$ on $K$ is likewise continuous there. 
Daniell has a theorem to the effect that a function equal for every argument to the greater or to the less of two summable functions is itself summable. The function which is the constant $H$ over the part of $C$ within $K$ and 0 elsewhere may be shown without difficulty to belong to $T_{1}$, and hence to be summable. Hence the function obtained from $W_{1}$ by replacing its values within $K$ by $A+\varepsilon$ or $B-\varepsilon$ is in fact summable.

I have so far been unable to eliminate without any further restriction the condition that $W_{1}$ be bounded, although I suspect that this condition is superfluous. The results obtained in this section are valid, of course, if for the word "circle" is substituted " $n$-sphere", in a space of $n$ dimensions.

\section{Certain summable functions}

A surface $M$ in $n$-space is said to have capacity $c$ if there is a positive function $f(P)$ defined over $M$ such that

$$
1=\left(\int_{M}\right)^{n-1} f(P)(P Q)^{n-2} d S
$$

(in the case $n=2$.

$$
\left.1=-\int_{M} f(P) \log P Q d S\right)
$$

independently of $Q$ so long as $Q$ remains on $M$, while

$$
\left(\int_{\mathbb{M}}\right)^{n-1} f(P) d S=c .
$$

A set of points on $C$ is said to have zero measure if the function which is 1 over the set and 0 over the rest of $C$ is summable, and determines a harmonic function 0 over $R$. I say that if we have given a closed set of points $S$ on the boundary $C$ of an $n$-dimensional region $K$ for which the Dirichlet problem is solvable, and if $S$ can be included in the interior of a surface $M$ of arbitrarily small capacity, then $S$ is of zero measure.

To prove this, let us remark that we can distribute over the part of $C$ within $M$ a boundary potential that is continuous, 1 on $S$, and 0 on the boundary of $M$. This boundary potential, together with the boundary potential 0 over the rest of $C$, will determine a harmonic function $u(Q)$ over $R$, which will be uniformly less, within $R$ and outside $M$, than 


$$
\left(\int_{M}\right)^{n-1} f(P)(P Q)^{n-2} d S
$$

or its two-dimensional analogue.* Now let $Q$ be a point of $R$ at a distance $a$ from the nearest point of $C$. Then (7) and hence $u(Q)$ will not exceed $c \dot{a}^{n-2}$ or $-c \log a$, as the case may be. Since $C$ may be made arbitrarily small, the $\dot{I}_{Q}$ of our boundary function that is 0 except on $S$, where it is 1 , cannot exceed 0 . It follows at once that $S$ is of zero measure. Thus a finite set of points, or, more generally, an $(n-2)$-spread on the $(n-1)$-spread bounding a region in the space of $n$ dimensions is of zero measure.

A function which is bounded and has discontinuities only at a closed set of points of zero measure is summable. Let $S$ be this set. Let $M_{a}$ be the set of all points on $C$ within a distance of less than $a$ from some point of $S$. Let $U(P)$ be any function bounded on $C$ and with all its discontinuities on $S$. It is then possible to form a function $U_{a}(P)$ continuous over the whole of $C$ and differing from $U(P)$ only over $M_{a}$.t It is even possible to keep the set of functions $\left\{U_{a}\right\}$ uniformly bounded. These functions $U_{a}$ remain summable even if any finite change is made in them over $S$, since any function finite over $S$ and zero over the rest of $C$ is less in absolute value than a function with Daniell integral everywhere zero, and hence is itself of zero Daniell integral. As $a$ approaches 0 , these functions $U_{a}$ thus modified can be made to approach $U$ boundedly over all $C$. Hence by a theorem of Daniell which we have already quoted, $U_{a}$ is summable.

* In the two-dimensional case, to avoid difficulties due to the change of sign of the logarithm, the unit of measurement should be chosen greater than the greatest linear dimension of $C$.

f Given any set of points, it is possible to surround it within any assigned distance by a polyhedral boundary. It is hence possible to surround the set by a sequence of such boundaries, each including the next, approaching to the set within any assigned distance. Given any function uniformly continuous on the original set, it is possible to approximate to it by continuous functions on the polyhedral boundaries which all lie between the bounds of the original function, and which all constitute a function continuous over the set of points consisting of the original set, together with the polyhedra. The continuous functions thus obtained may be taken as the boundary values of an infinite set of harmonic functions defined between the successive polyhedra and outside the outermost one. Together these constitute a continuous function extending the original continuous function through the whole of space, and a fortiori over any surface in space.

\section{MassachusetTs Institute of Technology,} Cambridge, Mass. 